\title{
THE EFFICACY BACKWARD WALKING AND SOFTTISSUE MANIPULATION IN PLANTAR FASCIITIS
}

Pradeep Shankar*1, Renuka Devi. M ${ }^{2}$, Dirk Labuscher ${ }^{3}$ Shameer Ali 4 Mohamed Elbaz ${ }^{4}$.

${ }^{* 1}$ Physiotherapist, Medeor Hospital , Abu Dhabi, UAE.

${ }^{2}$ Associate professor, JSS college of physiotherapy, Mysore, INDIA.

${ }^{3}$ Head of Physiotherapy, M edeor Hospital, Abu Dhabi, UAE.

${ }^{4}$ Orthopedic surgeon, M edeor Hospital , Abu Dhabi, UAE.

\section{ABSTRACT}

Background: Plantar fasciitis one of the most common foot pain condition treated by health care providers. Several physical therapy intervention applied to overcome heel pain but recent studies shown soft tissue manipulation is more effective in reducing pain.

Objective: One of the path mechanics behind plantar fasciitis is cyclic overload of plantar fascia occurs due to increased plantar flexion moment because of increased Achilles tendon tension and increased dorsiflexion moment of forefoot occurs due to increase ground reaction on forefoot while walking forward. This abnormal force will tend to be higher in plantar fasciitis patients make difficulty in walking and create more trigger point and stiffness in gastrocnemius muscle. To overcome this imbalance, assumption made Backward walking may be effective as it replicates lower limb movements mirror of forward walking and having advantage in reducing ground reaction force and reverse the plantar flexion moment in hind foot and dorsiflexion moment in forefoot. Study proposed to find its effective along with soft tissue manipulation in Plantar fasciitis.

Materials and M ethods: Six male subjects who has been diagnosed as Plantar Fasciitis with mean age 40 years referred from Orthopedic surgeon were taken as subjects in the study. Parameter VAS and Dorsi flexion range of ankle noted prior to and following 8 session of intervention. All subjects were randomly divided in two group $A$ and $B$ with three subject in each. Group $A$ underwent treatment of M yofascial and trigger point release technique on gastrocnemius muscles. Group B underwent same M yofascial and trigger point release technique as group $A$ in addition to it they underwent Backward walking (BW) on treadmill with $\% \%$ inclination

Outcome measures; Visual Anolog Scale (VAS), Ankle Dorsiflexion range.

Result: There was significant improvement in reduction of pain and increase in ankle Dorsiflexion Range of motion in group B than Group A .

Conclusion: Backward walking along with M yofascial release and trigger point release technique is found to be effective in reducing pain and to improve function in plantar fasciitis.

KEY WORDS: Backward walking, trigger point release technique, M yofascial release technique.

Address for correspondence: Mr. Pradeep Shankar, Physiotherapist , M edeor Hospital , Abu Dhabi, UAE. E-M ail: pradeerenu1921@gmail.com

\begin{tabular}{|c|c|c|}
\hline \multicolumn{3}{|c|}{ Access this Article online } \\
\hline \multirow{2}{*}{$\begin{array}{c}\text { Quick Response code } \\
\text { Dol: } 10.16965 \text { lijpr.2016.211 }\end{array}$} & \multicolumn{2}{|c|}{$\begin{array}{l}\text { International Journal of Physiotherapy and Research } \\
\text { ISSN } 2321-1822 \\
\text { www.ijmhr.org/ijpr.html }\end{array}$} \\
\hline & $\begin{array}{l}\text { Received: 30-12-2016 } \\
\text { Peer Review: 31-12-2016 } \\
\text { Revised: 06-01-2017 }\end{array}$ & $\begin{array}{l}\text { Accepted: 28-01-2017 } \\
\text { Published (0): 11-02-2017 } \\
\text { Published (P): 11-02-2017 }\end{array}$ \\
\hline
\end{tabular}

\section{BACKGROUND}

Plantar fasciitis one of the most common foot pain condition treated by health care providers [1]. Systemic review reported that plantar 
fasciitis accounts for between $8 \%$ and $15 \%$ of foot complaints in nonathletic and athletic populations. Patients with plantar heel pain usu-ally report insidious sharp pain under the heel, along the medial border of the plantar fascia to its insertion at the me-dial tuberosity of the calcaneus, upon weight bearing after a period of non-weight bearing. The pain is worse in the morning, with the first steps after getting out of bed, after prolonged periods of in-activity (eg, sitting), or at the beginning of a workout. The pain typically lessens with increasing activity (eg, walking, run-ning) but tends to worsen toward the end of the day. In some patients, these symp-toms can induce considerable functional limitations and prolonged disability [2].

Both surgical and nonsurgical ap-proaches have been proposed for the management of plantar heel pain.Clini-cal practice guidelines and the Cochrane Review have concluded that there has been limited evidence for the effective-ness of corticosteroid therapy, conflicting evidence for low-energy extracorporeal shockwave therapy, and no evidence for therapeutic ultrasound or low-intensity laser, in reducing pain in individuals with plantar heel pain. Among nonsurgical in-terventions, stretching of the gastrocne-mius muscle and the plantar fascia have shown moderate evidence of effectiveness for the management of plantar heel pain, although only in the short term [2]. Clear-ly, more studies are in need.

Simons et al have suggested that taut bands myofascial/muscle trigger points (TrPs) in the gastrocnemius muscles may be involved in the development of plantar heel pain [3]. TrPs are defined as hyperirrita-ble areas associated within a taut band of a skeletal muscle that are painful on com-pression, contraction, or stretching of the muscles, and elicit a referred pain distant to the $\operatorname{TrP}[3,4]$ The clinical reasoning for developing taut band in the gastrocne-mius muscle and its correlation on pathomachanics in Plantar fasciitis condition is as follows, it can be noted that the more tension in Achilles tendon occurs during weight bearing conditions like walking. The tension was more due to increased plantarflexion moment at the hind foot. This increase in plantarflexion moment create more the ground reaction forces on the forefoot and also the more dorsiflexion moments of the forefoot to hind foot. It is the combination of hindfoot plantarflexion moments and forefoot dorsiflexion moments that increase the tendency to arch flattening and collapse in midfoot joints. These collapsing forces are counteracted passively by tension of the plantar fascia. Plantar fascia tension in weightbearing conditions produce dorsiflexion moments of the hind foot to forefoot and plantarflexion moments of the forefoot to hind foot as a passive mechanical response for the increase in Achilles tendon tension and ground reaction forces in the forefoot. Conversely, a decrease of Achilles tendon tension reduces the plantarflexion moments of the hind foot, the amount of ground reaction forces in the forefoot, and the passive tension of plantar fascia during weight-bearing activities [5]. So it is very important to prevent or avoid plantar flexion moment at hind foot and more ground reaction force at fore foot during weight bearing stance of gait to reduce cyclic overload on plantar fascia. This cannot be achieved just by stretching of gastrocne-mius muscle. However Retrowalking or Backward walking(BW) replicates movements of lower limb mirror of forward walking may counteract this imbalance was assumed. Few studies on Backward walking kinetic and kinematic analysis like Grasso et al. did a study to find the difference in the origin of propulsion by ELECTRO M YOGRAPHIC analysis in Forward and Backward walking and found the main Forward walking (FW) propulsion is generated by the ankle plantarflexors, the principal BW propulsion is provided by the hip and knee extensors [6]. Naoki Soda etal found backward walking kinetic factors of ankle power and workload were lower during backward walking than in forward walking [7]. Considering these above factor study was conducted to find the efficacy of myofascial release and trigger point release technique on gastrocnemius muscles and backward walking in plant fasciitis .

\section{MATERIALS AND METHODS}

Participants: Six male patients with mean age 40, who has been diagnosed as Plantar Fasciitis 
with VISUAL ANOLOG SCALE(VAS) score $8 / 10$ referred from Orthopedic surgeons of M edeor Hospital were taken as subjects in the study . The subject who had plantar fasciitis along with associated disorder like ankle sprains, Osteoarthritis of knee and lumbar disc lesion with sciatica were excluded in this study. The purpose of study was explained and informed consent was obtained from all subjects.

Outcome measures: The outcome measures used in the study were VISUAL ANALOG SCALE [VAS] and Ankle Dorsiflexion range of motion of Ankle.

Intervention; All subjects base line restricted Ankle Dorsiflexion range of motion was measured with goniometry scale and noted. All subjects were randomly divided in two group $A$ and $B$ in three each.

Group A underwent treatment of Myofascial and trigger point release technique on gastrocnemius muscles. Subjects were examined and noted Active trigger point on the medial aspect Gastronemius muscle where Trigger point pressure release technique applied to patient. The pressure was maintained until the therapist per-ceived release of the taut band holding it to 90 second and repeated 3 times. Myofascial release technique Lifting or rolling technique on gastronemeus muscle belly and stretch strokes application done at Achilles tendon.

Group B underwent same M yofascial and trigger point release technique as group $A$, in addition to it they underwent Backward walking on treadmill with $0 \%$ inclination.Treadmill kept on normal flat or $0 \%$ level inclination and speed gradually increased to 1.5 miles per hour maintained for 5 minutes for first 3 session and increased to a duration 10 minutes on the following next 5 session. Intervention applied at the rate 3 times per week for 8 session. Self stretching application to gastrocnemius and plantar fascia extending great toe had been applied to all subject for 4 times with stretch hold for 10 second following intervention. This self stretching was taught as home exercise program to all subject and been advice to do early morning after getting up from bed and night before sleeping.

VAS and Ankle Dorsiflexion range was measured and noted at the end of $8^{\text {th }}$ session of treatment.

\section{RESULT}

The pre and post intervention data of parameter VAS and Ankle dorsiflexion range following were statistically analysed using students T test with baseline level of significance kept as 0.05 . software SPSS were used for statistic analysis.

Table1: Comparison of Group A versus B on parameter VAS score.

\begin{tabular}{|c|c|c|c|c|c|}
\hline & $\begin{array}{c}\text { PRE } \\
\text { Intervention } \\
\text { mean }\end{array}$ & $\begin{array}{c}\text { Post } \\
\text { Intervention } \\
\text { mean }\end{array}$ & $\begin{array}{c}\text { Standard } \\
\text { deviation }\end{array}$ & t value & $\begin{array}{c}\text { Significant } \\
\text { ( 2-tailed) }\end{array}$ \\
\hline Group A & 8 & 7 & 0 & 0 & \\
\hline Group B & 8 & 1 & 1 & 12.124 & 0.007 \\
\hline
\end{tabular}

Group A the correlation an t value cannot be computed because the standard error of difference is 0 .

Table 2: Comparison of Group A versus B on Dorsi flexion Range of motion of Ankle.

\begin{tabular}{|c|c|c|c|c|c|}
\hline & $\begin{array}{c}\text { PRE } \\
\text { Intervention } \\
\text { mean }\end{array}$ & $\begin{array}{c}\text { Post } \\
\text { Intervention } \\
\text { mean }\end{array}$ & $\begin{array}{c}\text { Standard } \\
\text { deviation }\end{array}$ & t value & Significant \\
\hline Group A & 10.6 & 8 & 0.57735 & 8 & 0.015 \\
\hline Group B & 11 & 2.3 & 0.57735 & 26 & 0.001 \\
\hline
\end{tabular}

Group B shown significant reduction in VAS score with t value 12.124 and $p \varangle 0.007$.

Group B shown significant increase in ankle dorsiflexion than Group A with $p \varangle 0.001$.

Simons et al (1999) advocated Gastronemius muscle trigger point as the potential source of heel pain[3,4]. Considering this, a therapeutic intervention on release of trigger point in gastronemieus muscle is applied in the study.

The possible hypothesis on relation of taut band, pain and stiffness in muscle could be, taut band reduce length of sarcomere and creates more stiffness to surrounding muscles and pain. By applying the triggerpoint release technique on taut bands it equalizes length of sarcomere, reduces stiffness and also the pain.

Others suggested that pain relief from TrP pressure may result from reac-tive hyperemia within the TrP Ajimsha et al (2014) and Shah (2014) advocated M yofascial release technique on gastronemeus and soleus in plantar fasciitis pain and found significant improvement in reducing pain and improvement in function than 
ultrasound therapy $[8,9]$. The myofascial release technique applied on gastronemius muscle medial and on achilis tendon might had assisted in reducing the adhesion between muscle fiber , fascia and tendon with muscle fiber attachment , assisted in increase in nutrition may assisted in decreasing the pain and to improve range of motion of dosiflexion of ankle.

According to Javier Pascual Huerta etal Patho mechanics and relation of gastronemius in plantar fasciitis asfollows, in static weight bearing conditions Achilles tension produces a plantarflexion moment at the ankle joint without movement as long as the foot remains in static equilibrium. This plantarflexion moment at the hind foot decreases ground reaction forces in the calcaneus and increases ground reaction forces in the forefoot displacing the center of pressure of the foot anteriorly to the forefoot.

The increase in ground reaction forces in the forefoot produces an increase of ankle joint dorsiflexion moments that counteract the plantarflexion moment at the ankle joint created by the increase in Achilles tendon tension. If the dorsiflexion moment produced by the ground reaction forces of the forefoot is of the same magnitude as the plantarflexion moment produced by the Achillestendon tensional force, the ankle joint will not move and will stay in static equilibrium. The increase in ground reaction forces in the forefoot also produces an increase in dorsiflexion moments of the forefoot to the hind foot at the midfoot joints The combination of hind foot plantarflexion moments and forefoot dorsiflexion moments created by Achilles tendon tension tends to flatten the arch of the foot. This flattening force on the arch is counteracted by tension of the plantar structures, such as the plantar fascia that produces a dorsiflexion moment of the hind foot to forefoot and a plantarflexion moment of the forefoot. Although there are several plantarstructures to avoid arch flattening, cadaveric studies have consistently shown that the plantar fascia is more important to prevent arch collapse than other [10].

The plantar fascia resists the combined downward compressive force of the body weight and the corresponding upward ground reaction force by virtue of its osseous connection. The plantar fascia undergoes a stretch tension from these forces, preventing flattening of the medial longitudinal arch, thus elevating the arch [10].

During the propulsive phase of gait, dorsiflexion of the toes wind the plantar fascia around the head of the metatarsal. This winding shortens the plantar fascia and elevates the medial longitudinal arch while creating a varus position of the calcaneus that locks the talonavicular and calcaneocuboid joints into stabilizing the midfoot. So it is very important to to prevent or avoid plantar flexion moment at hind foot and more ground reaction force at fore foot during weight bearing stance of gait to reduce cyclic overload on plantar fascia. This cannot be reduced by normal forward walking. In the present study we applied Backward walking, the patterns of movements in the joints of lower limb in backward walking is the reverse of forward walking.

According to Nokia soda et al (2013) found Ankle power during forward walking plantar flexion caused by the non-contractile extensibility (utilizing elastic energy) of the Achilles tendon acting as a spring. However, the plantar flexion power of the ankle during backward walking was low even when the angle of dorsal flexion prior to plantar flexion was large. This indicates that the influence of noncontractile elastic energy on plantar flexion is small. the propulsive force in backward walking must come from some factor other than the ankle. Possible sources of the propulsive force are compensation through hip power and the center of gravity.[7] Grasso et al. found that the difference in the origin of propulsion by EM G analysis, the main Forward walking(FW) propulsion is generated by the ankle plantarflexors, the principal BW propulsion is provided by the hip and knee extensors [6]. According to M inhyeon Lee etal (2013) In $\mathrm{BW}$, the ankle dorsiflexes after the toe-strike and then plantarflexes during most of the support phase In the ankle joint, the maximum plantarflexion moment was generated during loading response during BW. In this phase, the ankle decelerated to play an important part in shock absorption; thus, the maximum plantar flexion moment and the greatest power absorption occurred. According to several previous 
studies the plantarflexor muscles, the gastrocnemius and soleus, are activated in this phase. These muscles are activated in order to decelerate the ankle and absorb power. In midstance, the ankle joint plantarflexed to move the trunk backward, causing the greatest power generation during BW [11] . According to Grasso et al. reported that the main thrust is provided by knee and hip extensors during BW [6].These above studies support the present study and correlates with the result of study, where pain has decreased significantly than group $A$ due to reduce in plantar flexion moment at hind foot (decrease achilis tendon tension)and ground reaction force at fore foot during weight bearing in Backward walking. This is due to reduced propulsive force and increased power absorption of plantar surface of foot . Range also improved to significantly in ankle dorsiflexion due to more stretch of plantar flexor during backward walking.

Limitation: The study was conducted on limited subjects of six and taken only male subject with mean age of 40 . Base line VAS score all subjects were 8 . Parameter only VAS and Ankle Dorsiflexion were taken in the study.

\section{CONCLUSION}

Backward walking along with myofascial release and trigger point release technique in gastronemius muscle is effective in reducing pain and improve functional activity in plantar fasciitis.

Future scope of study: future studies should include larger population, its long term effect should be consider.

\section{ACKNOWLEDGEMENTS}

I acknowledge this study to Dr. Lalu Chacko, clinical head and medical director of Medeor hospital Abu Dhabi and M r. Safeer Ahmed, Head of M edeor hospital , Abu Dhabi for their support to my study. I acknowledge staff of orthopaedic and Physiotherapy department of Medeor hospital, Abu Dhabi for their support always.

\section{Conflicts of interest: None}

\section{REFERENCES}

[1]. Alvarez-Nemegyei J, Canoso JJ. Heel pain: diagnosis and treatment, step by step. Cleve Clin J Med. 2006;73:465-471.

[2]. Neufeld SK, Cerrato R. Plantar fasciitis: evalu-ation and treatment. J Am Acad Orthop Surg. 2008;16:338346.

[3]. Simons DG, Travel JG, Simons LS. Myofascial Pain and Dysfunction: The Trigger Point M anual; Volume 1 The Upper Half of Body. 2nd ed. Balti-more, MD: William and Wilkins; 1999.

[4]. Simons DG. Understanding effective treatments of myofascial trigger points. J Bodywork Mov Ther. 2002;6:81-8

[5]. Tao K, Ji WT, Wang DM, et al. Relative contributions of plantar fascia and ligaments on the arch static stability: a finite element study. Biomed Tech (Berl) 2010;55:265-71.

[6]. Grasso R, Bianchi L, Lacquaniti F. M otor patterns for human gait: backwardversus forward locomotion. Journal of Neurophysiology 1998;80:1868-85.

[7]. Naoki Soda. Three-dimensional M otion Analysis of the Ankle during Backward Walking. J. Phys. Ther. Sci.2013;25:747-749.

[8]. Shah H. A study on effect of myofascial release in plantar fasciitis. IJPOT;2014:21:261-66.

[9]. Ajimsha MS, Binsu D, Chithra S. Effectiveness of myofascial release in the management of plantar heel pain: a randomized controlled trial. Foot (Edinb).; 2014: 24:66-71.

[10]. Javier Pascual Huerta, PhD. The Effect of the Gastrocnemius on the Plantar Fascia. Article in Foot and Ankle Clinics of North America - December 2014. https:// www. researchgate. net/publication/ 266148170

[11]. M inhyeon Lee. Kinematic and kinetic analysis during forward and backward walking. Gait Posture (2013). Elsevier Publication.

[12]. RÔM ULO RENAN-ORDINE. Effectiveness of Myofascial Trigger Point $M$ anual Therapy Combined With a Self-Stretching Protocol for the M anagement of Plantar Heel Pain: A Randomized Controlled Trial. Journal of orthopaedic \& sports physical therapy 2011;41(92).

How to cite this article:

Pradeep Shankar, Renuka Devi. M , Dirk Labuscher, Shameer Ali , M ohamed Elbaz. THEEFFICACY BACKWARD WALKING AND SOFT TISSUE M ANIPULATION IN PLANTAR FASCIITIS. Int] Physiother Res 2017;5(1):1872-1876. DOI: 10.16965/ijpr.2016.211 